\section{AB0870 METABOLIC AND CARDIOVASCULAR GOUT ARE THE MOST FREQUENT CLINICAL VARIANTS OF THE DEBUTING GOUT}

E. Mikhnevich ${ }^{1}$, T. Pavlovich ${ }^{1}$, E. Mytnik ${ }^{2} .{ }^{1}$ Department of Internal Medicine, University of Medicine; ${ }^{2}$ Diagnostic Center, Rheumatology Clinic, Minsk, Belarus

Background: Gout is phenotypically and genotypically heterogeneous. In most cases, renal mechanism plays an important role in the development of gout. At the same time, gout, as a rule, occurs on the basis of pre-existing comorbid pathology.

Objectives: To determine clinic-pathogenetic variants of gout depending on a leading comorbid pathology at the gout onset

Methods: We included 604 patients with confirmed gout from our Center database. During 2010-2016, comorbidities were registered before the gout onset, at its appearance, during and at the last examination. Metabolic syndrome (MS) was defined by 5 components. CVD included CHD, atrial fibrillation, CHF and stroke. Patients after organ transplantation and with terminal renal failure observed in specialized centers were not recruited.

Results: In 67,1\% ( $\mathrm{n}=405)$ of patients, the gout developed on the basis of pre-existing MS, thus, this variant may be called a metabolic gout (MG) and considered as a component of MS. The patients with MG were younger, the mean age of symptoms onset was $42(35-48)$ years. At the first gout attack, all patients had $\mathrm{BMI} \geq 25 \mathrm{~kg} / \mathrm{m}^{2}, 8,2 \%(\mathrm{n}=33)$ had pre-existing $\mathrm{HTN}$, and in $14,3 \%(\mathrm{n}=58)$ of cases the diagnosis of HTN was firstly made. No one patient had diabetes mellitus (DM). Frequent regular or single abundant alcohol intake together with overeating as factors more often favoring gout attack were revealed in all patients. At the last examination, only $38,1 \%(n=230)$ of patients had MS without any CVD, the mean age was $50(45-55)$ years; $98,7 \%(n=227)$ had $\mathrm{BMl} \geq 25 \mathrm{~kg} / \mathrm{m}^{2} ; 73,9 \%$ $(n=170)$ presented with HTN; $59,1 \%(n=136)$ showed dyslipidemia; 6,5\% $(n=15)$ had DM. In $8,3 \%(n=19)$ of patients, changing the lifestyle and normalizing the weight resulted not only in decreased hyperuricemia but lower attacks rate or even their total disappearance during a 5 year follow-up. The second variant of gout onset developing in patients with pre-existing CVD, can be called a CV gout or cardiorenovascular (CRV) gout. This type of gout was revealed in $28,5 \%$ $(n=172)$ of patients, the disease debuted later, at the age of $60(55-64)$ years $(p<0,001) .97,7 \%(n=163)$ had HTN $\left(x^{2}=30,1, p<0,001\right)$ and $27,3 \%(n=47)-$ DM $\left(x^{2}=32,65, p<0,001\right)$, both features were more common in CV gout. $85,5 \%$ $(n=147)$ had $B M l \geq 25 \mathrm{~kg} / \mathrm{m}^{2}(F=0,066, p<0,001)$. Regular alcohol intake was considerably lower $-33,7 \%(n=58)\left(x^{2}=167,4, p<0,001\right)$, but medication use was higher - diuretics in $14,5 \%(n=25)\left(x^{2}=21,77, p<0,001\right)$ and low-dose aspirin in $37,2 \%(n=64)$ of patients $(F=0,24, p<0,001)$. At the last examination, the mean age of patients was 63 (59-69) years $(p<0,001)$, chronic GFR $<60 \mathrm{ml} / \mathrm{min}$ was observed in $47,7 \%(n=82)$ of patients $(F=0,34, p<0,001)$.

The secondary gout developed in $4,4 \%(n=27)$ of patients with various diseases: myelo-, lymphoproliferative and other blood diseases - 6 cases, renal gout - 13, gut gout after partial gut resection - 2, and anorexic gout - 2. Alcoholic gout without MS was diagnosed in 4 patients, all men with body mass deficiency.

Conclusions: In our opinion, there are two main clinic-pathogenetic variants of debuting gout - metabolic and cardiovascular, the both with different conditions of occurrence, progression and prognosis. In clinical practice, it is reasonable to differentiate these variants, that is determined by the need to simplify complex multimodal diagnostic and therapeutic approach to the patients with gout.

Disclosure of Interest: None declared

DOI: 10.1136/annrheumdis-2017-eular.3524

\section{AB0871 NSAIDS AND LIVER FUNCTION IN PATIENTS WITH GOUTY ARTHRITIS}

E. Mikhnevich ${ }^{1}$, T. Rayeuneva ${ }^{1}$, K. Leonchik ${ }^{1}$, E. Mytnik ${ }^{2} .{ }^{1}$ University of Medicine; ${ }^{2}$ Rheumatology Clinic, Diagnostic Center, Minsk, Belarus

Background: Nonsteroidal anti-inflammatory drugs (NSAIDs) have been associated with hepatotoxicity. Drug-induced liver injury (DILI) in patients under NSAIDs is more frequently presented by hepatocellular damage with elevated serum alanine aminotransferase (ALT) levels (1).

Objectives: To evaluate the hepatocellular toxicity due to NSAIDs on the basis of changes in serum ALT levels in patients with gouty arthritis (GA).

Methods: 376 patients with GA registered in our database were included in our retrospective study. Males made $92,8 \%(n=349)$. Mean age was $57(50-62)$ years; mean duration of gout $-7(3-10)$ years. NSAIDs were prescribed to the patients with gouty attack by generalists, and then all patients were addressed to the Rheumatology Clinics. Serum ALT levels were determined at the onset of gout attack and 3-10 days after starting NSAIDs therapy. All NSAIDs used were generics. The hepatotoxicity was considered when ALT level was 2 times (or more) upper limits of normal values (42 U/I for men and $35 \mathrm{U} / \mathrm{l}$ for women) according to DILI criteria. ALT level at baseline was within the normal limits in $80,8 \%(n=304)$ of cases, its elevation was identified in $19,2 \%(n=72)$.

Results: We divided the patients into 3 groups according to the type of changes in serum ALT levels after receiving NSAIDs:

Group 1: $69,4 \%(n=261)$ of patients with normal ALT $24(17-30) \quad U / I$ before and 24 (18-32) U/l after treatment $(p>0,05)$

Group 2: $11,4 \%(n=43)$ of patients with normal basal ALT $26(21-35) \mathrm{U} / \mathrm{l}$ and its elevation to $77(72-88) \mathrm{U} / \mathrm{l}(\mathrm{p}<0,001)$ after NSAIDs therapy.

Group 3: $19,2 \%(n=72)$ of patients with elevated basal ALT 57 (46-91) U/l, who had different type of ALT response to NSAIDs therapy. We identified 3 subgroups within the third group:

Subgroup A: $7 \%(n=5)$ of patients with elevated ALT level at the beginning of gout attack - $92(62-123,5) \mathrm{U} / \mathrm{l}$ and higher value after treatment - $151(129-215)$ $\mathrm{U} / \mathrm{l}(\mathrm{p}<0,001)$. Subgroup B: $23,6 \%(n=17)$ of patients with elevated ALT before treatment - $53(47,5-62) \mathrm{U} / \mathrm{l}$ and non-significant changes after therapy - 51 $(44,5-63,5) U / /(p>0,05)$. Subgroup C: $69,4 \%(n=50)$ of patients with elevated ALT before treatment - 59 (49-89) U/l and reduced and/or normal ALT level after therapy - $42(29-57) U / l(p<0,001)$. While the patients from subgroup $A$ have demonstrated supplementary hepatotoxicity due to NSAIDs on the basis of pre-existing liver injury, the absence of changes in ALT levels in subgroup B has been suggested in favor of a chronic liver injury. Subgroup $C$ has reflected the effect of NSAIDs to decrease and even normalize the elevated basal ALT value. This may be linked to a hepatic inflammation, which is likely associated with alcohol intake, but hyperuricemia could also be involved.

Conclusions: $69,4 \%$ (261 from 376 ) of patients with GA having normal basal ALT values did not demonstrate any changes in ALT level after NSAIDs treatment; in $11,4 \%$ of patients, hepatocellular NSAIDs-induced hepatotoxicity was revealed. In the group of patients with elevated basal ALT level, the response of ALT to NSAIDs treatment was as follows: no significant changes $-23,6 \%$, elevated value $-7 \%$, reduced or normalized value $-69,4 \%$. The last subgroup $(C)$ requires more detailed study.

\section{References:}

[1] Schmeltzer P. A., Kosinski A. S., Kleiner D. E. et al. Liver injury from nonsteroidal anti-inflammatory drugs in the United States. Liver International 2016;36(4): 603-609.

Disclosure of Interest: None declared

DOI: 10.1136/annrheumdis-2017-eular.3542

\section{AB0872 DIAGNOSTIC EFFICIENCY OF DETERMINING THE PURINE METABOLISM ENZYMES ACTIVITY IN THE DIFFERENTIATION OF GOUT}

E. Mozgovaya, A. Trofimenko, S. Bedina, I. Zborovskaya. Federal State Budgetary Institution "Research Institute of Clinical and Experimental Rheumatology", Volgograd, Russian Federation

Background: Serum uric acid (UA) level is the most important risk factor for gout. At the same time many people with hyperuricemia do not suffer from gout, and UA level can return to normal during gout exacerbation.

Objectives: to improve the diagnostics of the gout.

Methods: 53 gout patients were the target group. The mixed control group was consisted of 150 rheumatoid arthritis patients, 95 osteoarthritis patients, 55 ankylosing spondylitis patients. The diseases were diagnosed in accordance with the international standards. Adenine deaminase, Adenosine deaminase (ADA), AMP-deaminase (AMPDA), Guanine deaminase, Guanosine deaminase, Guanosine phosphorylase, 5'-nucleotidase, Xanthin dehydrogenase (XDG), Xanthin Oxidase, Purine nucleoside phosphorylase activities were determined in blood serum with subsequent evaluation of sensitivity (Se), specificity (Sp), likelihood ratio of positive (LRP) and, likelihood ratio of negative (LRN) result, predictive value of positive and negative results.

Results: The AMFDA and ADA activities were defined as a screening marker to diagnose of the gout from a joint syndrome another genesis. Threshold AMPDA activity 1,67 IU was characterized with the predictive value of negative results (without taking into account the prevalence) near $100 \%$, Se $98 \%$, Sp $62,45 \%$.

Cutoff value (CV) of ADA activity $(9,57 \mathrm{IU})$ was characterized with LRN 0,104, Se $92,45 \%$, Sp $72,65 \%$, the sum (Se+Sp 165,10\%), which was maximum not only for this test but for all the studied enzymes. The predictive value of negative results (without taking into account the prevalence) was $98,20 \%$. The clinically significant CV of positive result for the ADA activity definition was identified also: LRP was 6,93 (Se 22,64\%, Sp 96,73\%) for the range of $12.58 \mathrm{IU}$ and above. This fact allows the use of this test not only for the screening, but also, in some cases, for the verification of gout with significant pretest probability of this disease.

Two CV were selected for the XDG activity: 7,49 $\mu \mathrm{M} / \mathrm{l} \times \min$ (Se 47,17\%, Sp $97,14 \%$ ) and $8,39 \mu \mathrm{M} / \mathrm{I} \times \min (\mathrm{Se} 39,62 \%$, Sp $99,59 \%)$. The result of XDG activity determining in the range of $7.49-8.39 \mu \mathrm{M} / 1 \times \min$ is an important argument in favor of the gout presence, but this may be useful for differential diagnosis only at high pretest probability of the gout.

Conclusions: Determination of the ADA activity was gave way to AMPDA activity determination at the gout screening and to XDG activity determination at gout verification. However, the ADA multifunctionality, evidenced clinically significant presence of the second CV (positive results) and a small proportion of the cases, which covered a "grey area" between CV $(27.48 \%)$, provided the ability to use this marker as a backup in the absence of diagnostically relevant AMPDA and XDG activities.

Disclosure of Interest: None declared

DOI: 10.1136/annrheumdis-2017-eular.2304 